Results It is established that the nephroblastoma increases blood flow to the main artery of the affected kidney compared with the contralateral ( $p=0,00003$ ). According to our data the volume of the affected organ with nephroblastoma correlated with blood flow in his main artery $(r=0.45 ; \mathrm{p}<0.05)$. Such dependence can reflect the pathological circulation of the affected kidney. It also reflects the need to change the absolute amount of blood flow in tumor growth. At the same time in a healthy kidney specific blood flow much higher than that of the affected $(p=0,00001)$. Indices of relative blood flow affected and contralateral kidneys were 1.32 (1.04-2.13) $\mathrm{ml} / \mathrm{cm}^{3} / \mathrm{min}$ and $5.46(3.73-6.78) \mathrm{ml} / \mathrm{cm}^{3} / \mathrm{min}$, respectively.

Conclusions Thus, the hemodynamic characteristics the affected of nephroblastoma kidney were studied. Specific blood flow of affected organ with a continuous functional activity is significantly less with respect to the contralateral (not affected). These data may prove useful in the development of differential diagnostic criteria for distinguishing nephroblastoma and adrenal neuroblastoma at the stage of diagnosis.

\section{BLOOD PRESSURE AND HEART RATE DO NOT REFLECT CARDIAC OUTPUT IN CRITICALLY ILL CHILDREN}

doi:10.1136/archdischild-2012-302724.0779

'A Nusmeier, ${ }^{2} \mathrm{PJ}$ Gooskens, 'JG van der Hoeven, ' IJ Lemson. 'Intensive Care, Radboud University Nijmegen Medical Center, Nijmegen; ${ }^{2} T e c h n i c a l$ Medicine, University of Twente, Enschede, The Netherlands

Introduction Treatment to support the circulatory state is often based on the interpretation of clinical parameters while advanced hemodynamic monitoring is not always available or applied in children. Cardiac output(CO) measurement using the transpulmonary thermodilution(TPTD) technique is the gold standard in pediatric patients. We studied the predictive value of clinical parameters of (changes in) $\mathrm{CO}$ by comparing the blood pressure and heart rate values with intermittent $\mathrm{CO}_{\text {TPTD }}$ measurements.

Methods A retrospective observational study was performed in a heterogenic critically ill pediatric patient population. Doses of inotropics, if administered, was registered to study their influence on trend monitoring. $\mathrm{CO}$ was compared with heart rate(HR) and mean arterial blood pressure(MAP) one minute before every CO measurement. The results were analyzed using correlation and linear regression statistics.

Results 216 CO measurements in 20 patients were analyzed. Median age was 1.7 (range 0.3-5.2) years and median body weight 8.8 (range $3.8-18$ ) kg. The median CO was 1.88 (range 0.61-5.64)1/ min. The correlation coefficient (Spearman's rho) between the CO and HR and MAP was $-0.08(p<0.24)$ resp. 0.31 $(p<0.0001)$. Linear regression analysis of $\mathrm{CO}$ and both $\mathrm{HR}$ and $\mathrm{MAP}$ and the influence of inotropics on the relation of changes in $\mathrm{CO}$ and changes in $\mathrm{HR}$ and MAP are shown in table 1.

Conclusions Heart rate and blood pressure are unreliable in predicting cardiac output in critically ill children. The use of inotropics seems to increase the correlation between changes in HR and BP and changes in $\mathrm{CO}$.

Abstract 779 Table 1 Linear regression analysis

\begin{tabular}{|c|c|c|c|c|}
\hline dopendent varibblo & Indopendentyziallas & sumplecine. & $\pi$ & cienilisance lovel \\
\hline co (all) & HR, MAP & 215 & 0.29 & $<0,001$ \\
\hline Aco (alli) & $\triangle(H R, M A D)$ & 215 & 0.37 & $<0.001$ \\
\hline$\triangle C O(+$ inotropics) & $\triangle(M, D, M A P)$ & 119 & 0.45 & 00.001 \\
\hline$\Delta C O$ - inotropiss) & $\triangle(H E, M A D)$ & 97. & 0.27 & $<0.031$ \\
\hline
\end{tabular}
NEVUS SYNDROME IN A CHILD

doi:10.1136/archdischild-2012-302724.0780

F Alsohime. Pediatric Nephrology, Hopital Femme Mere Enfant, Lyon, France
Epidermal nevus syndrome is a rare congenital sporadic neuroectodermic disorder, characterized by the presence of epidermal nevi in association with various developmental abnormalities of the skin, eyes, nervous, skeletal, cardiovascular and urogenital systems. We describe a 5-year-old boy with conjunctival lipodermoid, cervical and facial sebaceous nevi who presented at 3 years of age with hypertension due to bilateral renal artery stenosis together with multiple vascular anomalies (aorta, celiac trunk, superior mesenteric artery) as shown by magnetic resonance angiography. Systemic arterial hypertension was difficult to control despite combined anti-hypertensive drugs and the surgical repair of the aortic coarctation.

\section{SEEKING FOR DEFINITIONS OF POOR PERFUSION STATES (PPS) IN LOW BIRTH WEIGHT INFANTS (LBWI) (PART II)}

doi:10.1136/archdischild-2012-302724.0781

'MC Bravo, 'P López-Ortego, 'L Sánchez, ${ }^{2} \mathrm{~J}$ Riera, ${ }^{1} \mathrm{~F}$ Cabañas, ${ }^{3} \mathrm{E}$ Pérez-Fernández, 1J Quero, 'A Pellicer. 'La Paz University Hospital, Department of Neonatology; ${ }^{2 B i o-}$ Engineer and Nanotechnology Department, Polytechnic University of Madrid; ${ }^{3}$ Division of Statistics, La Paz University Hospital, Madrid, Spain

Background and Aims No validated scoring system to diagnose PPS in LBWI is available. Echocardiography (Echo)-derived low superior vena cava flow (SVCF) is a biomarker of PPS in this population as associates adverse outcome. We examined the ability of clinical surrogates of low systemic blood flow to indicate PPS as defined by low SVCF.

Methods One hundred LBWI [27.4 (2) wks; 1014 (316) g] who reached disease score below threshold, underwent early $(<12 \mathrm{~h})$ and serial Echo scans during the first $96 \mathrm{hs}$ after birth. Mean blood pressure $(\mathrm{MBP})$, lactate, base excess $(\mathrm{BE})$, core-to- peripheral temperature gap (DT) and diuresis were registered at the time of Echo assessment. N-terminal probrain natriuretic peptide (NT-proBNP) and troponine were measured within $24 \mathrm{~h}$ and at postnatal day 4 .

Results No association between SVCF and MBP, lactate, BE, DT, diuresis or troponine was found. NT-proBNP was inversely related to SVCF ( $p=0.006)$. Low SVCF $(<41 \mathrm{~mL} / \mathrm{k} / \mathrm{min})$ was present in the first Echo (4.2h) in 27 patients. At that time, the sensitivity and specificity of the clinical parameters to predict low SVCF was, respectively: lactate $>4.5 \mathrm{mmol} / \mathrm{L}(22.2 \%$; 89.4\%); BE <-9 $(6.9 \%$; $92.9 \%) ; \mathrm{MBP}<30 \mathrm{mmHg}$ (25\%; 64.6\%); DT (15\%; 78.8\%). Combination of lactate and BE did not improve accuracy.

Conclusions PPS is a common condition early after birth in the sick LBWI. The role of Echo-derived systemic blood flow assessment to identify PPS cannot be replaced by clinical assessment solely.

Disclaimer Supported by the Spanish Health Ministry and the SAMID network.

\section{NEW DILUTION METHOD FOR OP/OS MEASUREMENT IN PATIENTS WITH SINGLE VENTRICLE (SV) ANATOMY}

doi:10.1136/archdischild-2012-302724.0782

N Krivitski, V Kislukhin, N Thuramalla, A Kriksunov. Transonic Systems Inc., Ithaca, NY, USA

Background and Aims Major challenge for treatment of Hypoplastic left heart syndrome by Norwood procedure is in achieving the adequate $\mathrm{ap} / \mathrm{Qs}$ value. The absence of routine method of assessing the Op/Os value can lead to hypoxia, brain injury, for $\mathrm{Op} / \mathrm{Q} s<<$ 1 or to insufficient tissue perfusion and lung edema for $a_{p} / Q_{s}>>1$. The aim of the study was to develop routine method for $\mathrm{Op} / \mathrm{Os}$ assessment for PICU and NICU patients.

Method development: A mathematical model of indicator movement for SV anatomy was developed. After intravenous injection and mixing in SV the first portion of the indicator enters systemic 
circulation via aorta. Second portion of the indicator enters lungs via $\mathrm{PA}$, then again enters SV via left atria etc. The model suggests that $\mathrm{Op} / \mathrm{Q}$ s may be calculated from dilution curve (Pic.1) Op/ Os=S2/S1.

Results COstatus monitor, (Transonic Systems Inc., NY, USA) was used in NICU and PICU patients to measures cardiac output, blood volumes and to identify shunts and PDA. According to Transonic curve data archive recoded by COstatus for single ventricle patients the actual shape of dilution curves (example, Pic.2) well agrees with model data.

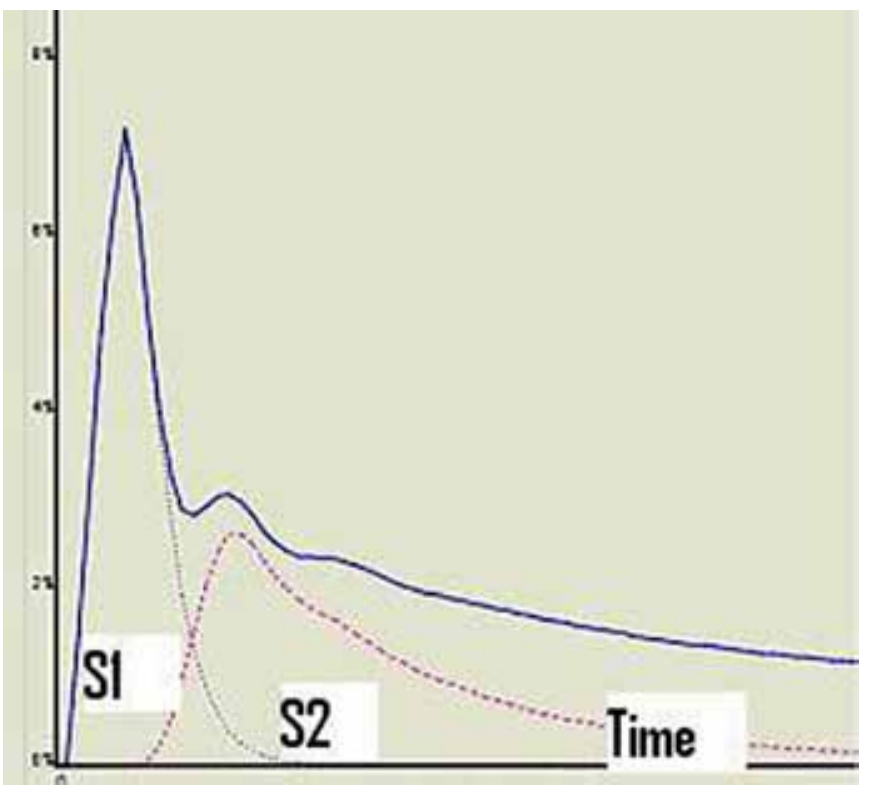

Abstract 782 Figure 1 Dilution Curve Model

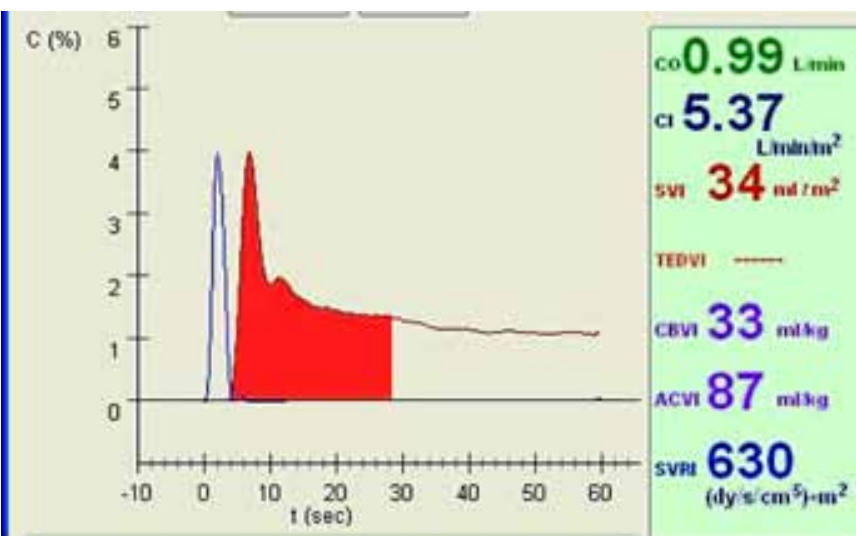

Abstract 782 Figure 2 SV Patient Dilution Curve

Conclusions Mathematical model for indicator movement in SV anatomy proved that $\mathrm{Op} / \mathrm{Os}$ value can be calculated from indicator dilution curve. Next step is to validate the $\mathrm{ap} / \mathrm{Q}$ s values measured by COstatus in animal model and in patients.

Grant NIH SBIR \# R43 HL111852-01.

\section{MANAGEMENT OF SEVERE PAEDIATRIC TRAUMA BRAIN INJURY (PTBI) GUIDED BY INTRACRANIAL PRESSURE (ICP) MONITORING IN A PICU IN ALGERIA}

doi:10.1136/archdischild-2012-302724.0783

H Bouguetof, MA Negadi, K El Halimi, D Boumendil, ZC Mentouri. Pediatric Intensive Care Unit, Faculty of Medicine - Oran University, Oran, Algeria
Background and Aims PTBI is the leading cause of death and long-term morbidity. Current recommendations for the management of severe PTBI (Glasgow Coma [GCS] score $\leq 8$ ) indicate that ICP monitoring is appropriate in infants and children (Option). The most reliable methods of ICP monitoring are ventricular catheters and intra parenchymal systems. The aim of this study is to evaluate the management of PTBI based on continues monitoring of intraparenchymal ICP in a PICU in Algeria.

Methods Between January 2005 and December 2009 we collected 308 PTBI, 57 patients had intraparenchymal ICP monitoring. The consensus is to treat ICP exceeding the $20 \mathrm{mmHg}$ threshold, and to optimize cerebral perfusion pressure (CPP).

Results The mean age was 8 years, hypoxia and hypotension were frequent at admission, median GCS after resuscitation $=6$, ICP monitoring was set up by the intensivist in the PICU after un average time of 13 hours after trauma. Intracranial hypertension was detected and treated (mannitol, hyerventilation and thiopental) in more than $90 \%$ of cases. the average time of ICP monitoring was 5 days. No complications (infection, hemorrhage) with this technique was detected.

Conclusion The etiology and the pathophysiology of raised ICP in PTBI is a complex challenge for the intensivist. CPP and ICP were the first brain-specific targets for goal-directed therapies enacted in PTBI. In this study, ICP monitoring allows to detect intracranial hypertension and guide treatment better than when this technique is absent even if it is not a standard of the recommendations.

\section{SEEKING FOR DEFINITIONS OF POOR PERFUSION STATES (PPS) IN LOW BIRTH WEIGHT INFANTS (LBWI) (PART I)}

doi:10.1136/archdischild-2012-302724.0784

'MC Bravo, 'P López-Ortego, 'L Sánchez, 2J Riera, 'F Cabañas, E Pérez-Fernández, 'J Quero, 'A Pellicer. 'La Paz University Hospital, Department of Neonatology; ${ }^{2}$ BioEngineer and Nanotechnology Department, Polytechnic University of Madrid; ${ }^{3}$ Division of Statistics, La Paz University Hospital, Madrid, Spain

Background and Aims Echocardiography-derived low superior vena cava flow (SVCF) associates intraventricular haemorrhage, neurodisability and death. The weaknesses of the method relate to its variability. We aim to explore the relationship between two SVCF cut-off values to define PPS in LBWI and the patients' shortterm neonatal co-morbidities.

Methods One hundred LBWI [27.4 (2) wks; 1014 (316) g] who reached illness score below threshold, underwent early $(<12 \mathrm{~h})$ and serial echocardiography for the first $96 \mathrm{hs}$ after birth. The primary outcome was low SVCF prevalence according to two thresholds: < $41 \mathrm{ml} / \mathrm{k} / \mathrm{min}$ and $[<41 \mathrm{ml} / \mathrm{k} / \mathrm{min}$ + SVCF repeatability index (RI)] ( $\mathrm{RI}$ is twice the standard deviation of the differences divided by the mean of all the measures). Secondary outcomes were short-term neonatal clinical outcomes in relation to SVCF status.

Results $\mathrm{SVCF}<41 \mathrm{ml} / \mathrm{k} / \mathrm{min}$ prevalence was $30 \%$ and was associated with immaturity $(\mathrm{p}=0.02)$, corioamnionitis (0.007), advanced resuscitation at birth (0.004), lower Apgar scores $(\mathrm{p}<0.01)$ and postnatal ischemic events (bowel perforation or arterial vasospasm) $(\mathrm{p}=0.002)$. At SVCF $<51 \mathrm{ml} / \mathrm{k} / \mathrm{min}(41 \mathrm{ml} / \mathrm{k} / \mathrm{min}+$ repeatability index) cut-off value, the PPS prevalence was $50 \%$; in addition to the above-mentioned co-morbidities trends showed an association between PPS and combined adverse outcome (death or intracranial haemorrhage).

Conclusions Low SVCF is highly prevalent in the sick LBWI during the early postnatal period. The association of low SVCF with ischemic events and adverse outcome supports this biomarker as an indicator of PPS.

Disclaimer No conflict of interest. Study supported by the Spanish Health Ministry, SAS/2481/2009, the SAMID network (RD08/0072/0018). 